\title{
DISCRETE TRIANGULATED CATEGORIES
}

\author{
NATHAN BROOMHEAD, DAVID PAUKSZTELLO, AND DAVID PLOOG
}

\begin{abstract}
We introduce and study several homological notions which generalise the discrete derived categories of D. Vossieck. As an application, we show that Vossieck discrete algebras have this property with respect to all bounded t-structures. We give many examples of triangulated categories regarding these notions.
\end{abstract}

\section{CONTENTS}

1. Properties of triangulated categories: cone finite, hom bounded 2

2. Discreteness with respect to t-structures 4

3. Derived-discrete algebras $\Lambda(r, n, m) \quad 7$

4. Discreteness with respect to co-t-structures 9

5. Examples

\section{INTRODUCTION}

In this article, we investigate Hom-finite triangulated categories which are, in various senses, small. Our motivating examples are bounded derived categories of derived-discrete algebras, which were introduced and classified by D. Vossieck [28]. In previous work [13], we observed some special properties of these categories: the dimension of Hom spaces between indecomposables is bounded (by 1 or 2, depending on the algebra), and all hearts of bounded t-structures have only finitely many indecomposable objects.

We set out to introduce and compare abstract notions which apply to such examples. The three most relevant for this article are

- cone finite: any two objects admit only finitely many cones, up to isomorphism;

- hom bounded: universal bound on Hom dimension among indecomposable objects;

- countable: the category has only countably many objects, up to isomorphism.

We establish the following relations between these properties in Theorem 1.2:

Theorem. (i) A hom 1-bounded triangulated category is cone finite.

(ii) A cone finite triangulated category with a classical generator is countable.

We give examples showing that countability doesn't imply cone finite or hom bounded, and that cone finite doesn't imply hom bounded. Vossieck's definition of discreteness does not generalise to abstract triangulated categories, as it invokes cohomology objects, i.e. a t-structure. Therefore, we study pairs $(\mathrm{D}, \mathrm{H})$ of a triangulated category together with the heart of a bounded t-structure. Moreover, the Grothendieck group $K_{0}(\mathrm{D})=$ $K_{0}(\mathrm{H})$ enters, generalising dimension vectors of modules. We introduce two notions characterising different aspects of smallness for abelian categories via their K-groups which we call modular and abelian discrete (see Section 2), and we prove in Theorem 2.5:

Theorem. Let $(\mathrm{D}, \mathrm{H})$ be a triangulated category with the heart of a bounded $t$-structure.

(i) $\mathrm{D}$ cone finite and $\mathrm{H}$ abelian discrete $\Longrightarrow \mathrm{D}$ is discrete with respect to $\mathrm{H}$

(ii) $\mathrm{D}$ is discrete with respect to $\mathrm{H} \Longrightarrow \mathrm{H}$ abelian discrete.

(iii) $\mathrm{D}$ is discrete with respect to $\mathrm{H}$ and $\mathrm{H}$ modular $\Longrightarrow \mathrm{D}$ cone finite.

MSC 2010: 18E30, 16G10, 16E35 
As one application of this result, we show that derived-discrete algebras are discrete with respect to any bounded t-structure, not just the standard one; see Proposition 3.2.

In Section 4, we introduce and study an analogous definition of discreteness with respect to a bounded co-t-structure with co-heart C, assuming the existence of a silting object.

There are many interesting examples of triangulated categories having certain of the properties in question. Here, we list some of them. For a more elaborate version, with further properties and example classes, see the table on page 12. Below, $\Gamma_{\mathrm{ADE}}, A_{\infty}$ and $\tilde{A}_{1}$ denote any $\mathrm{ADE}$ quiver, the one-sided infinite quiver of type $A$ with zigzag orientation, and the Kronecker quiver, respectively. The column then refers to the bounded derived category of the path algebra. DDC stands for the bounded derived category of a deriveddiscrete algebra $\Lambda(r, n, m)$ with $r<n$, i.e. of finite global dimension. Finally, $\mathrm{T}_{w}$ is the triangulated category generated by a $w$-spherical object.

\begin{tabular}{lcccccccc}
\hline & $\mathrm{k} \Gamma_{\mathrm{ADE}}$ & $\mathrm{k} A_{\infty}$ & $\mathrm{DDC}$ & $\mathrm{T}_{1}$ & $\mathrm{~T}_{>1}$ & $\mathrm{~T}_{<1}$ & $\mathbb{Q} \tilde{A}_{1}$ & $\mathbb{F}_{q} \tilde{A}_{1}$ \\
\hline H-discrete & $\checkmark$ & $\checkmark$ & $\checkmark$ & $\checkmark$ & $\checkmark$ & - & $\times$ & $\checkmark$ \\
C-discrete & $\checkmark$ & $\checkmark$ & $\checkmark$ & - & - & $\checkmark$ & $\times$ & $\checkmark$ \\
hom bounded & $\checkmark$ & $\checkmark$ & $\checkmark$ & $\times$ & $\checkmark$ & $\checkmark$ & $\times$ & $\times$ \\
cone finite & $\checkmark$ & $\checkmark$ & $\checkmark$ & $\checkmark$ & $\checkmark$ & $\checkmark$ & $\times$ & $\checkmark$ \\
countable objects & $\checkmark$ & $\checkmark$ & $\checkmark$ & $\checkmark$ & $\checkmark$ & $\checkmark$ & $\checkmark$ & $\checkmark$ \\
finite hearts & $\checkmark$ & $\times$ & $\checkmark$ & $\times$ & $\checkmark$ & - & $\times$ & $\times$ \\
\hline
\end{tabular}

We mention other approaches for capturing the smallness of categories and algebras:

Small Krull-Gabriel (KG) dimensions of (the abelianisations of) triangulated categories correspond to "small" categories. For example, by work of G. Bobiński and H. Krause [11], the KG dimension of (perfect categories) of Dynkin quivers is 0, and that of derived-discrete algebras is 1 if the algebra has infinite global dimension and 2 otherwise. However, there are non-derived-discrete algebras whose perfect categories have KrullGabriel dimension 2; for the example of the Kronecker quiver, see [25, Proposition 1.8].

Another abstract concept for triangulated categories is that of a generic object, and its absence, generic triviality. In [19], Z. Han shows that generic triviality of a compactly generated triangulated category is equivalent to local finiteness of the compact subcategory, and to the compact subcategory having KG dimension 0. Thus it seems unlikely that these notions are useful in the study of the smallness notions investigated here.

In $[1,27]$ the authors study discreteness of triangulated categories related to finiteness of intervals of t-structures and silting objects. They apply this to contractibility of spaces of stability conditions.

In [18], Y. Han and C. Zhang characterise derived-discrete algebras as the finitedimensional algebras of finite global cohomological length. Their approach depends on cohomology and modules, i.e. does not apply to abstract triangulated categories.

Acknowledgments: It is a pleasure to thank Martin Kalck, Henning Krause, Greg Stevenson for their input and suggestions, as well as an anonymous referee. Moreover, we thank Mike Prest and The University of Manchester for their hospitality. We are grateful to the London Mathematical Society for financial support via their 'Research in Pairs' Scheme 4 grant, no. 41434. The second named author was supported by EPSRC grant no. EP/K022490/1.

\section{Properties of triangulated CAtegories: CONE Finite, Hom Bounded}

We define a number of properties that suitable $\mathbf{k}$-linear triangulated categories can enjoy, all of which capture certain aspects of 'smallness'. Throughout, we assume that the 
class of objects of any category forms a set. Moreover, we apply the following abuse of terminology: whenever we speak of a 'set' of objects defined by some property, we mean the class of such objects, up to isomorphism.

Fix a field k. A k-linear category is called Hom-finite if all homomorphism spaces are finite-dimensional over k. An additive category is called Krull-Schmidt if each object has a decomposition into a finite direct sum of indecomposable objects. The decomposition is unique up to isomorphism and re-ordering of the summands. Examples are Hom-finite abelian categories and their bounded derived categories.

Throughout this note we shall write $\operatorname{hom}(A, B)=\operatorname{dim} \operatorname{Hom}(A, B)$.

Definition 1.1. Let D be a Hom-finite, Krull-Schmidt k-linear triangulated category.

(1) $\mathrm{D}$ is called cone finite if for any two objects $D_{1}, D_{2} \in \mathrm{D}$, the set of cones of morphisms $D_{1} \rightarrow D_{2}$, i.e. the set $\left\{C \in \mathrm{D} \mid \exists D_{1} \rightarrow D_{2} \rightarrow C \rightarrow \Sigma D_{1}\right\}$, is finite.

(2) $\mathrm{D}$ is called hom b-bounded for some $b \in \mathbb{N}$ if $\operatorname{hom}\left(D_{1}, D_{2}\right) \leq b$ for any indecomposable objects $D_{1}, D_{2} \in$ ind(D). The minimal such $b$ is called the hom bound of $\mathrm{D}$, and $\mathrm{D}$ is called hom bounded if it is hom $b$-bounded for some $b \in \mathbb{N}$.

(3) D is called countable, if the set of all objects up to isomorphism is countable. Equivalently, ind(D) is a countable set, as D is Krull-Schmidt and Hom-finite.

All of these definitions could be stated in greater generality: cone finiteness makes sense for all triangulated categories (no field needed); hom boundedness applies to arbitrary k-linear categories (no triangulated structure required); countability of objects applies to arbitrary categories. The latter notion is crude, and depends strongly on the cardinality of the field $\mathbf{k}$; see Remark 1.3. We will not explore these properties beyond the setting of Hom-finite triangulated categories.

In this article, we also study the relationship with Vossieck's notion of discreteness, see Section 2, and we introduce and investigate its co-t-structure counterpart in Section 4. For now, we only deal with the above three notions: they have the advantage of applying in a general setting, i.e. without additional data. Also note each condition (countable objects, hom bounded, cone finite) is automatically passed on to triangulated subcategories.

Theorem 1.2. $\quad$ (i) A hom 1-bounded triangulated category is cone finite.

(ii) A cone finite triangulated category with a classical generator is countable.

Proof. (i) Suppose that D is hom bounded with bound 1, i.e. $\operatorname{hom}(A, B) \leq 1$ for all $A, B \in \operatorname{ind}(\mathrm{D})$. In particular, this implies that nonzero morphisms $A \rightarrow B$ with $A, B \in$ ind(D) have isomorphic cones. Consider a morphism of the form

$$
A_{1} \oplus \cdots \oplus A_{n} \stackrel{\left(a_{1}, \ldots, a_{n}\right)^{t}}{\longrightarrow} B
$$

where $A_{1}, \ldots, A_{n}$ and $B$ are indecomposable. If one $a_{i}=0$, then a standard application of the octahedral axiom shows that the cone splits up as follows:

$$
A_{1} \oplus \cdots \oplus A_{n} \stackrel{\left(a_{1}, \ldots, a_{n}\right)^{t}}{\longrightarrow} B \longrightarrow C\left(\left(a_{1}, \ldots, a_{i-1}, a_{i+1}, \ldots a_{n}\right)^{t}\right) \oplus \Sigma A_{i} .
$$

Therefore, we can assume that all $a_{i} \neq 0$. Any other such morphism $A_{1} \oplus \cdots \oplus A_{n}$ is of the form $\left(\lambda_{1} a_{1}, \ldots, \lambda_{n} a_{n}\right)^{t}$ for scalars $\lambda_{1}, \ldots, \lambda_{n} \in \mathbf{k}$ and hence induces a commutative diagram of distingished triangles

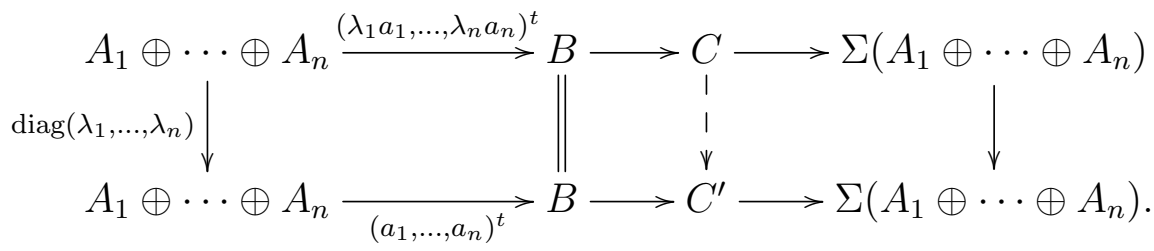


As above, we may assume that all $\lambda_{i} \neq 0$. But then both vertical morphisms in the left hand square are isomorphisms, hence the dashed arrow is also an isomorphism. It follows that there are finitely many possible cones $C$ for morphisms $A_{1} \oplus \cdots \oplus A_{n} \rightarrow B$.

Now consider the cone of an arbitrary morphism $\bigoplus_{i=1}^{n} A_{i} \rightarrow \bigoplus_{j=1}^{m} B_{j}$. We proceed by induction on $m$. For $m=1$, we are done above, so assume $m>1$. We have the following diagram coming from the octahedral axiom:

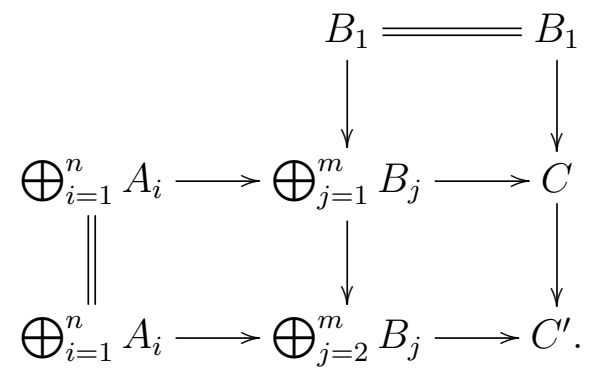

By induction, there are finitely many possible $C^{\prime}$. By the case for $m=1$ above, for each $C^{\prime}$ there are finitely many possible cones of $C^{\prime} \rightarrow \Sigma B_{1}$, and in particular, finitely many possibilities for $C$. Hence $\mathrm{D}$ is cone finite.

(ii) Recall that a classical generator of a triangulated category D is an object $G$ such that every object is obtained from $G$ in finitely many steps by taking shifts, cones and summands. (There does not have to be a bound on the number of steps; if such bounds exist, their minimum is the Rouquier dimension of D.) Bounded derived categories of finite-dimensional algebras and projective varieties have classical generators.

In each step, there are finitely many possibilities for summands by Krull-Schmidt; countably many possibilities for sums; and finitely many possibilities for cones by the assumption. Therefore, with $G$ generating $\mathrm{D}$ in countably many steps, the cardinality of objects of $\mathrm{D}$ is countable as well.

Remark 1.3. (1) The assumption of a classical generator in the theorem cannot be dropped: if $\mathrm{D}$ is any cone finite triangulated category, and $I$ some uncountable set, then $\bigoplus_{I} \mathrm{D}$ is still cone finite but uncountable.

(2) The same proof shows a bit more: if D has a classical generator and for all $A, B \in \mathrm{D}$, there are only countably many cones of morphisms $A \rightarrow B$, then $\mathrm{D}$ is countable. In particular, this applies to any Hom-finite $\mathbf{D}$ if $\mathbf{k}$ is a countable field.

(3) We remark that if $\mathbf{k}$ is a finite field, then D Hom-finite trivially implies cone finite. Hence, for fields of arbitrary cardinality, hom boundedness captures 'smallness' best among the notions of Definition 1.1. See the table in Section 5.

Conjecture 1.4. Hom bounded triangulated categories are cone finite.

\section{Discreteness With Respect to T-Structures}

In this section we consider an abstracted version of Vossieck's [28] original definition of derived-discrete algebras. Examining the derived categories of these algebras in [13] was our motivation to introduce the categorical notions in this article.

A torsion pair in a triangulated category $D$ consists of full subcategories $(X, Y)$, each of which is closed under taking direct summands, such that $\operatorname{Hom}(X, Y)=0$ and

$$
\mathrm{D}=\mathrm{X} * \mathrm{Y}:=\{D \in \mathrm{D} \mid \exists X \rightarrow D \rightarrow Y \rightarrow \Sigma X \text { with } X \in \mathrm{X}, Y \in \mathrm{Y}\} .
$$

A torsion pair $(\mathrm{X}, \mathrm{Y})$ is bounded ${ }^{1}$ if $\bigcup_{n \in \mathbb{Z}} \Sigma^{n} \mathrm{X}=\mathrm{D}=\bigcup_{n \in \mathbb{Z}} \Sigma^{n} \mathrm{Y}$.

\footnotetext{
${ }^{1} \mathrm{Be}$ aware that this a different use of the word 'bounded' than the one from Definition $1.1(2)$, the property 'hom bounded' of triangulated categories. No confusion should arise.
} 
A t-structure is a torsion pair $(\mathrm{X}, \mathrm{Y})$ with $\Sigma \mathrm{X} \subseteq \mathrm{X}$. Any t-structure induces an abelian category, its heart $\mathrm{H}:=\mathrm{X} \cap \Sigma \mathrm{Y}$. To any t-structure in a triangulated category, there are associated cohomology functors, denoted $H^{i}: \mathrm{D} \rightarrow \mathrm{H}$ for $i \in \mathbb{Z}$.

Bounded t-structures can be reconstructed from their hearts. In this note, we only deal with bounded t-structures, and we will simply say 'bounded heart' to mean a full abelian subcategory which is the heart of a bounded t-structure. Background on t-structures can be found in, for example, $[22, \S 10.1]$.

Definition 2.1. Let D be a Hom-finite, Krull-Schmidt k-linear triangulated category admitting bounded t-structures.

(1) Let $\mathrm{H}$ be the heart of a bounded t-structure. Then $\mathrm{D}$ is said to be discrete with respect to $\mathrm{H}$, or $\mathrm{H}$-discrete, if for every group valued function $v: \mathbb{Z} \rightarrow K_{0}(\mathrm{D})$, the set of objects $\left\{D \in \mathrm{D} \mid\left[H^{i}(D)\right]=v(i) \in K_{0}(\mathrm{D}) \forall i \in \mathbb{Z}\right\}$ is finite.

(2) D is said to have finite hearts if the heart of any bounded t-structure in D has only finitely many indecomposable objects.

In (1), $v$ is not assumed to be a group homomorphism.

There is a well-known, canonical isomorphism of Grothendieck groups, $K_{0}(\mathrm{H})=K_{0}(\mathrm{D})$, induced by the inclusion $\mathrm{H} \hookrightarrow D$. The inverse is given by sending the class of a complex to the alternating sum of the classes of its cohomologies with respect to $\mathrm{H}$. Moreover, given a finite-dimensional k-algebra $\Lambda$ having $N$ (non-isomorphic) simple modules, there is a further canonical isomorphism $K_{0}(\Lambda):=K_{0}(\bmod (\Lambda))=\mathbb{Z}^{N}$, mapping the class of a module to its dimension vector.

Let us establish the link between the above definition of discreteness and Vossieck's original notion: in [28], he exclusively considers categories of the form $D=D^{b}(\Lambda)$ for finite-dimensional $\mathbf{k}$-algebras over an algebraically closed field $\mathbf{k}$. He calls the derived category $\mathrm{D}^{b}(\Lambda)$ of the algebra $\Lambda$ discrete if for any sequence $v: \mathbb{Z} \rightarrow K_{0}(\Lambda)$ with only finitely many nonzero terms, the set of isomorphism classes of indecomposable complexes $A \in \mathrm{D}^{b}(\Lambda)$ with dimension vector $\underline{\operatorname{dim}}(A):=\left(\underline{\operatorname{dim}}\left(H^{i}(A)\right)\right)_{i \in \mathbb{Z}}=v$ is finite. Using the standard isomorphisms $K_{0}\left(\mathrm{D}^{b}(\Lambda)\right)=K_{0}(\Lambda)=\mathbb{Z}^{N}$, it is clear that $\mathrm{D}^{b}(\Lambda)$ is discrete in Vossieck's sense if and only if $\mathrm{D}^{b}(\Lambda)$ is discrete with respect to $\bmod (\Lambda)$ in the above sense.

The finite hearts property came up in our previous work on spaces of stability conditions of derived-discrete algebras [14].

Because we have to work with hearts in triangulated categories, we now also introduce some notions that capture 'smallness' of abelian categories. Let $\mathrm{H}$ be a Hom-finite, klinear abelian category, then $\mathrm{H}$ is Krull-Schmidt [6]. We denote $\pi: \mathrm{Ob}(\mathrm{H}) \rightarrow K_{0}(\mathrm{H})$. Recall that we identify objects up to isomorphism. For an object $H \in \mathrm{H}$, we denote by $\operatorname{Sub}(H)$ the set of subobjects $H^{\prime} \hookrightarrow H$, and by $\operatorname{Fac}(H)$ the set of factors $H \rightarrow H^{\prime}$.

- $\mathrm{H}$ is a length category if it is artinian and noetherian.

- $\mathrm{H}$ is finite if the set ind $(\mathrm{H})$ is finite.

- $\mathrm{H}$ is (abelian) discrete if $\pi$ has finite fibres, i.e. for any $c \in K_{0}(\mathrm{H})$, the set of objects $\pi^{-1}(c)=\{A \in \mathrm{H} \mid[A]=c\}$ is finite.

- $\mathrm{H}$ is modular if $\pi(\operatorname{Sub}(H))$ is a finite set for all $H \in \mathrm{H}$, i.e. $\left\{\left[H^{\prime}\right] \mid \exists H^{\prime} \hookrightarrow H\right\} \subseteq$ $K_{0}(\mathrm{H})$ is finite.

Of these, length and modular are mild restrictions. For example, they hold for $\bmod (\Lambda)$ with $\Lambda$ a finite-dimensional algebra. The other two conditions (discrete and finite) are severe restrictions.

Remark 2.2. All hearts for derived-discrete algebras are representation-finite module categories by $[13, \S 7.1]$. Since, by an exercise in string combinatorics, the derived-discrete 
algebras not derived equivalent to $\Lambda(1, n, m)$ are also representation-directed (see [5, Ch. IX]), they therefore satisfy a stronger property than abelian discrete: the class of an indecomposable module determines the module uniquely.

We will justify the terminology 'modular' below in Remark 2.4. For now, just observe that the condition is equivalent to the finiteness of $\pi(\operatorname{Fac}(H))$, since $\left[H^{\prime \prime}\right]=[H]-\left[H^{\prime}\right]$ for any short exact sequence $0 \rightarrow H^{\prime} \rightarrow H \rightarrow H^{\prime \prime} \rightarrow 0$.

Lemma 2.3. Let $H^{\prime}, H^{\prime \prime}$ be objects of a modular abelian category $\mathrm{H}$. Then the $K_{0}$-classes of objects $H$ with exact sequences $H^{\prime} \rightarrow H \rightarrow H^{\prime \prime}$ are finitely determined by the classes $\left[H^{\prime}\right]$ and $\left[H^{\prime \prime}\right]$, i.e. the set $\left\{[H] \mid \exists H^{\prime} \rightarrow H \rightarrow H^{\prime \prime}\right.$ exact $\} \subseteq K_{0}(\mathrm{H})$ is finite.

Proof. The exact sequence $H^{\prime} \stackrel{f}{\rightarrow} H \stackrel{g}{\rightarrow} H^{\prime \prime}$ leads to short exact sequences

$$
0 \rightarrow \operatorname{ker}(f) \rightarrow H^{\prime} \rightarrow \operatorname{im}(f) \rightarrow 0 \quad \text { and } \quad 0 \rightarrow \operatorname{im}(g) \rightarrow H^{\prime \prime} \rightarrow \operatorname{coker}(g) \rightarrow 0 .
$$

As $\mathrm{H}$ is modular, $[\operatorname{im}(f)]$ is finitely determined by $\left[H^{\prime}\right]$, and $[\operatorname{im}(g)]$ is finitely determined by $\left[H^{\prime \prime}\right]$. Moreover, $[H]=[\operatorname{ker}(g)]+[\operatorname{im}(g)]=[\operatorname{im}(f)]+[\operatorname{im}(g)]$, from $0 \rightarrow \operatorname{ker}(g) \rightarrow H \rightarrow$ $\operatorname{im}(g) \rightarrow 0$. Thus, $[H]$ is finitely determined by $\left[H^{\prime}\right]$ and $\left[H^{\prime \prime}\right]$, as claimed.

Remark 2.4. The property of the lemma captures the positivity of dimension vectors for modules over a finite-dimensional algebra $\Lambda$. If $\Lambda$ has $N$ simple modules, then $K_{0}(\Lambda)=$ $\mathbb{Z}^{N}$, and the class of a module $M$ is encoded in its dimension vector $\operatorname{dim}(M) \in \mathbb{N}^{N}$.

All submodules have smaller dimension vectors, hence $\mathrm{H}:=\bmod (\Lambda)$ is a $\operatorname{modular}$ abelian category. The lemma generalises the inequality $\underline{\operatorname{dim}}(M) \leq \underline{\operatorname{dim}}\left(M^{\prime}\right)+\underline{\operatorname{dim}}\left(M^{\prime \prime}\right)$ for an exact sequence $M^{\prime} \rightarrow M \rightarrow M^{\prime \prime}$.

Moreover, over an algebraically closed field $\mathbf{k}$ the equivalence

$$
\bmod (\Lambda) \text { discrete } \Longleftrightarrow \bmod (\Lambda) \text { finite, i.e. } \Lambda \text { has finite representation type }
$$

holds by the validity of the second Brauer-Thrall conjecture; see for example [5, Ch. IV.5] and the references therein. In general, these notions are not equivalent. For example, tubes are Hom-finite hereditary abelian categories that are discrete but not finite.

Theorem 2.5. Let $(\mathrm{D}, \mathrm{H})$ be a triangulated category together with the heart of a bounded t-structure. Then

(i) D cone finite and $\mathrm{H}$ abelian discrete $\Longrightarrow \mathrm{D}$ is discrete with respect to $\mathrm{H}$

(ii) $\mathrm{D}$ is discrete with respect to $\mathrm{H} \Longrightarrow \mathrm{H}$ abelian discrete.

(iii) $\mathrm{D}$ is discrete with respect to $\mathrm{H}$ and $\mathrm{H}$ modular $\Longrightarrow \mathrm{D}$ cone finite.

Corollary 2.6. Let $(\mathrm{D}, \mathrm{H})$ be a triangulated category with a modular heart of a bounded t-structure. Then

$$
\mathrm{D} \text { is discrete with respect to } \mathrm{H} \Longleftrightarrow \mathrm{D} \text { cone finite and } \mathrm{H} \text { abelian discrete. }
$$

Proof. For an object $D \in \mathrm{D}$, we define the function $v_{D}: \mathbb{Z} \rightarrow K_{0}(\mathrm{D})$ by $v_{D}(i):=\left[H^{i}(D)\right]$. For a function $v: \mathbb{Z} \rightarrow K_{0}(\mathrm{D})$, we define:

- $\mathrm{D}_{v}:=\left\{A \in \mathrm{D} \mid\left[H^{i}(A)\right]=v(i) \forall i \in \mathbb{Z}\right\}$, a full subcategory;

- $\operatorname{supp}(v):=\{i \in \mathbb{Z} \mid v(i) \neq 0\}$, the support of $v$;

- length $(v):=\max _{i \in \mathbb{Z}}\{v(i) \neq 0\}-\min _{i \in \mathbb{Z}}\{v(i) \neq 0\}$, the length of $v$.

(i) Given $v$, we do induction on the length of $v$. If length $(v)=0$, then we can assume that $v(0) \neq 0$, by suspending if necessary. Then all objects of $\mathrm{D}_{v}$ have a single cohomology in degree 0 , hence are in the heart $\mathrm{H}$. Thus, $\mathrm{D}_{v}=\{A \in \mathrm{H} \mid[A]=v(0)\}$, and this set is finite by our assumption that $\mathrm{H}$ is abelian discrete. 
Now let length $(v)=n>0$. Again, without loss of generality, we can assume that $\operatorname{supp}(v) \subseteq\{0, \ldots, n\}$. Define $v^{\prime}, v^{\prime \prime}: \mathbb{Z} \rightarrow K_{0}(\mathrm{D})$ by $v^{\prime \prime}(i)=v(i)$ for $1 \leq i \leq n$ and zero otherwise; and $v^{\prime}(0)=v(0)$ and zero otherwise. By induction, the subcategories $\mathrm{D}_{v^{\prime}}$ and $\mathrm{D}_{v^{\prime \prime}}$ are finite. Now for any object $A \in \mathrm{D}_{v}$, the truncation triangle for $A$ with respect to $H^{0}$ has the form $A^{\prime} \rightarrow A \rightarrow A^{\prime \prime} \rightarrow \Sigma A^{\prime}$ with $A^{\prime} \in \mathrm{D}_{v^{\prime}}$ and $A^{\prime \prime} \in \mathrm{D}_{v^{\prime \prime}}$. Hence, $\mathrm{D}_{v} \subseteq \mathrm{D}_{v^{\prime}} * \mathrm{D}_{v^{\prime \prime}}$. However, as $\mathrm{D}$ is cone finite, there are only finitely many cones out of the finitely many objects from the two subcategories. Hence, $\mathrm{D}_{v}$ is also finite.

(ii) This is immediate: given $c \in K_{0}(\mathrm{H})$, define $v: \mathbb{Z} \rightarrow K_{0}(\mathrm{D})=K_{0}(\mathrm{H})$ by $v(0):=c$ and $v(i)=0$ for $i \neq 0$. Since $\mathrm{D}$ is discrete with respect to $\mathrm{H}$, the set of objects $D \in \mathrm{D}$ with $\left[H^{i}(D)\right]=v(i)$ for all $i \in \mathbb{Z}$ is finite. By construction, $H^{i}(D)=0$ for all $i \neq 0$, i.e. $D \in \mathrm{H}$, and $[D]=v(0)=c \in K_{0}(\mathrm{H})$. Hence $\mathrm{H}$ is abelian discrete.

(iii) For $A, B \in \mathrm{D}$, we want to show that there are only finitely many cones $A \stackrel{f}{\rightarrow} B \rightarrow C_{f}$, where $f \in \operatorname{Hom}(A, B)$ is arbitrary. Any such triangle gives rise to a long exact cohomology sequence in $\mathrm{H}$

$$
\cdots \rightarrow H^{i}(A) \rightarrow H^{i}(B) \rightarrow H^{i}\left(C_{f}\right) \rightarrow H^{i+1}(A) \rightarrow H^{i+1}(B) \rightarrow \cdots .
$$

By Lemma 2.3, $\left[H^{i}\left(C_{f}\right)\right] \in K_{0}(\mathrm{H})$ is determined up to finite ambiguity by $\left[H^{i}(B)\right]$ and $\left[H^{i+1}(A)\right]$. As the long exact sequence is finite (the t-structure is bounded), we see that all $\left[H^{i}\left(C_{f}\right)\right]$ are determined by $A$ and $B$, up to finite ambiguity (even more, they are determined by the functions $v_{A}, v_{B}$, but we do not need this). Hence, for fixed $A$ and $B$, there are only finitely many possibilities for $v_{C_{f}}$. Finally, since $\mathrm{D}$ is $\mathrm{H}$-discrete it follows that for each such choice of $v_{C_{f}}$, there are only finitely many objects $C_{f}$ realising this function. Altogether, the number of cones of morphisms $A \rightarrow B$ is finite.

We expect the following statements to hold in general. In the next section, we show that they do hold for derived-discrete algebras. Note that a triangulated category D can be discrete with respect to a bounded heart $\mathrm{H}$ which is hom unbounded; see the tube category $\mathrm{T}_{1}$ in the table on page 12 . This example also yields a bounded heart with infinitely many indecomposable objects.

Conjecture 2.7. Let $\mathrm{D}$ be a Hom-finite Krull-Schmidt triangulated category and $\mathrm{H}$ the heart of a bounded t-structure.

(i) If $\mathrm{D}$ is $\mathrm{H}$-discrete, then all bounded hearts in $\mathrm{D}$ are discrete.

(ii) If $\mathrm{D}$ is $\mathrm{H}$-discrete, then so is $\left(\mathrm{D}, \mathrm{H}^{\prime}\right)$ for any bounded heart $\mathrm{H}^{\prime}$.

(iii) If $\mathrm{D}$ is $\mathrm{H}$-discrete and $\mathrm{H}$ is finite, then all bounded hearts are finite.

(iv) $\mathrm{D}$ is $\mathrm{H}$-discrete $\Longleftrightarrow \mathrm{D}$ is cone finite.

\section{DeRiveD-Discrete AlgEBRAS $\Lambda(r, n, m)$}

Recall that in [28], a finite-dimensional algebra was defined to have a discrete derived category if $\mathrm{D}^{b}(\Lambda)$ is discrete with respect to $\bmod (\Lambda)$ in our sense, i.e. with respect to the standard heart. Following standard usage, we call such an algebra derived-discrete.

By the classification of G. Bobiński, C. Geiß and A. Skowroński [10], such an algebra is derived equivalent to either a representation-finite hereditary algebra or to the path algebra $\Lambda(r, n, m)$ given by a cycle of length $n$ to which a linearly oriented $A_{m}$-chain is attached; bound by $r$ consecutive zero relations in the cycle, ending at the trivalent vertex. Here, $m \geq 0,1 \leq r \leq n$. In the following, we assume that $r<n$, which is equivalent to $\Lambda(r, n, m)$ having finite global dimension. 


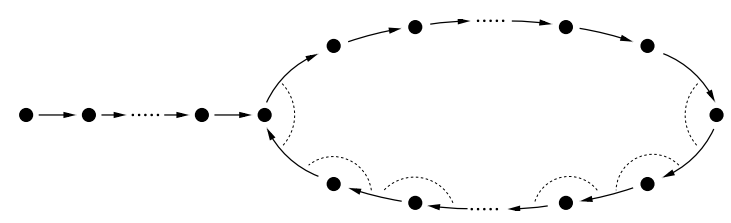

We mention two basic facts about these categories from our previous work:

Proposition 3.1 ([13, Theorem 5.1 and Proposition 6.1]). Let $\mathrm{D}=\mathrm{D}^{b}(\Lambda(r, n, m))$ be the bounded derived category of a derived-discrete algebra. Then

(i) If $r=1$, then $\mathrm{D}$ is hom 2-bounded. If $r>1$, then $\mathrm{D}$ is hom 1-bounded.

(ii) Hearts of bounded t-structures are finite.

We use this together with the results of Section 2 to show that discreteness for $\Lambda(r, n, m)$ is actually independent of the bounded t-structure.

Proposition 3.2. Let $\Lambda$ be a derived-discrete algebra. Then $\mathrm{D}^{b}(\Lambda)$ is discrete with respect to any bounded heart $\mathrm{H}$.

Proof. As $\bmod (\Lambda)$ is the module category of a finite-dimensional algebra (in particular modular) and $\mathrm{D}^{b}(\Lambda)$ is $\bmod (\Lambda)$-discrete, the triangulated category $\mathrm{D}^{b}(\Lambda)$ is cone finite by Theorem 2.5(iii). Moreover, any bounded heart $\mathrm{H}$ is finite by Proposition 3.1. Therefore, $\mathrm{D}^{b}(\Lambda)$ is discrete with respect to $\mathrm{H}$ by Theorem 2.5(i).

Propositions 3.1 and 3.2 show that Conjecture 2.7 holds for $\mathrm{D}^{b}(\Lambda(r, n, m))$. When $r>1$, these categories even enjoy a property slightly stronger than classical Vossieckdiscreteness, i.e. $\mathrm{H}$-discreteness with respect to the standard heart: classes of cohomology modules of indecomposable complexes are even unique. For this statement, we can relax the finite global dimension assumption and allow $n=r$.

Proposition 3.3. If $\Lambda$ is a derived-discrete algebra with $\mathrm{D}^{b}(\Lambda) \simeq \mathrm{D}^{b}(\Lambda(r, n, m))$ with $n \geq r>1$, then the indecomposable complexes $D$ in $\mathrm{D}^{b}(\Lambda)$ are uniquely determined by the sequences $\left[H^{i}(D)\right] \in K_{0}(\mathrm{D})$.

Proof. We explain the computation for $\Lambda:=\Lambda(r, n, m)$; the computation for a derivedequivalent presentation of $\Lambda$ is similar. Since $\Lambda$ is gentle, by [7] the indecomposable complexes in $\mathrm{K}^{b,-}(\Lambda)$ are given by (homotopy) string complexes; see [9] for the terminology and $[3, \S 2]$ for an overview. The string complexes for $\Lambda$ are listed in [3, Lemma 7.1]. Now [15, Theorem 2.8] describes the strings of the indecomposable summands of $H^{\bullet}(D)$ for a string complex $D$, which implies that $H^{i}(D)$ is either indecomposable or zero.

Using the orientations of the homotopy strings given in $[3, \S 7]$, one sees that the cohomology of each string complex over $\Lambda$ has an indecomposable module in its minimal degree, a (possibly trivial) periodic part, corresponding to the cycle, consisting of a repeating unique indecomposable module, and an indecomposable module in its maximal degree. In the infinite global dimension case, the periodic part has zero cohomology. In particular, each string complex is uniquely specified by its cohomology. The result then follows because each indecomposable $\Lambda$-module is uniquely determined by its dimension vector; see Remark 2.2 and recall $r>1$.

Example 3.4. In addition, discrete derived categories are not 'cone unique': there are indecomposable objects $A, B$ of $\mathrm{D}^{b}(\Lambda(1,2,1))$ and nonzero maps $f, g: A \rightarrow B$ having non-isomorphic cones. The pathology again only occurs in the case $r=1$; obviously such behaviour is impossible when $r>1$, as then Hom spaces between indecomposable complexes are 1-dimensional and lead to unique cones. 
Consider $\Lambda(1,2,1)$, i.e. the path algebra for the quiver $-1-a \rightarrow 0 \rightleftarrows b \longrightarrow 1$ bound by the zero relation $b c$ at the vertex 0 . Write $P:=P(-1)$ and $Q:=P(0)$ for the projective modules associated to vertices -1 and 0 , respectively.

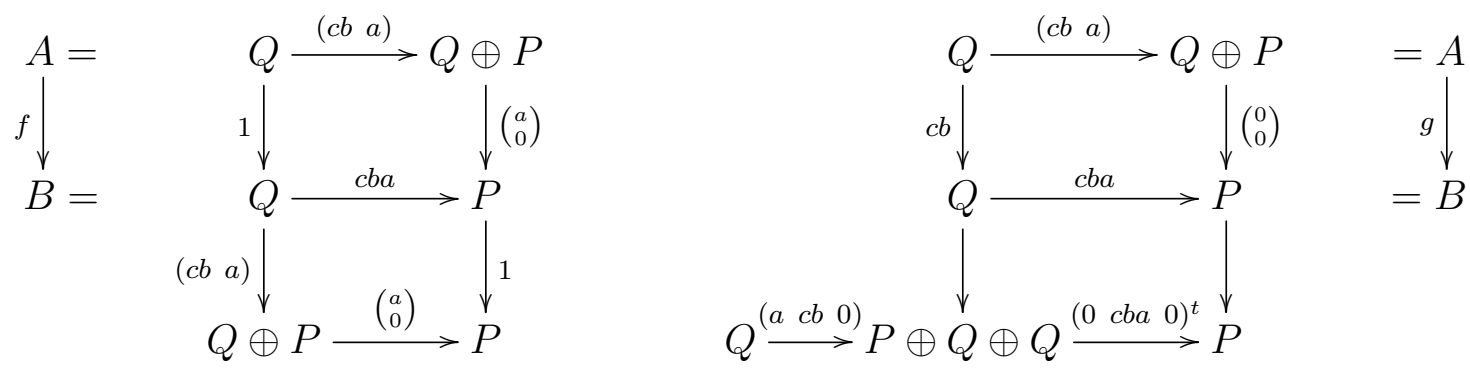

Then the cones are $(Q \rightarrow 0) \oplus P \rightarrow Q$ and $(0 \rightarrow Q \rightarrow 0) \oplus(P \rightarrow P \oplus Q \rightarrow Q)$. Above, all the matrices are transposed because, to match up with string combinatorics for these examples, we read compositions of maps from left to right.

Remark 3.5. We expect that all results of this section are also true for the deriveddiscrete algebras $\Lambda(n, n, m)$ of infinite global dimension. In fact, the hom bound was established in [3, Theorem 7.4].

Our results suggest the following question.

Question 3.6. Are derived-discrete algebras $\Lambda$ characterised, among finite-dimensional algebras, by $\mathrm{D}^{b}(\Lambda)$ having only finite bounded hearts?

Relation to compactly generated triangulated categories. We briefly discuss 'big' triangulated categories, i.e. assuming the existence of all set-indexed coproducts. Such categories are not Hom-finite, and hence outside the scope of the rest of this article.

Recall that a (compactly generated) triangulated category D is called pure-semisimple if each object $D \in \mathrm{D}$ is pure-injective; we refer the reader to [16, Section 2] for the definition of pure-injectivity in the setting of triangulated categories.

Let $\Lambda$ be a finite-dimensional algebra. By [8, Theorem 12.20], the category $\mathrm{D}=$ $\mathrm{D}(\operatorname{Mod}(\Lambda))$ is pure-semisimple if and only if $\Lambda$ is derived equivalent to a representationfinite hereditary algebra. However, by [4], each indecomposable object in the homotopy category $\mathrm{K}(\operatorname{Proj}(\Lambda(r, n, m)))$ is pure-injective but $\mathrm{K}(\operatorname{Proj}(\Lambda(r, n, m)))$ is not puresemisimple; similarly also for $\mathrm{D}(\operatorname{Mod}(\Lambda(r, n, m)))$. This raises the following question:

Question 3.7. Does the property that each indecomposable object of a big compactly generated triangulated category is pure-injective, characterise discreteness among big triangulated categories?

\section{Discreteness With Respect to CO-T-Structures}

A co-t-structure in a triangulated category $\mathrm{D}$ is a torsion pair $(\mathrm{X}, \mathrm{Y})$ such that $\Sigma^{-1} \mathrm{X} \subseteq \mathrm{X}$. Its co-heart is defined to be $\mathrm{C}:=\mathrm{X} \cap \Sigma^{-1} \mathrm{Y}$, which is an additive category that is, in general, not abelian.

Let $(D, C)$ be a Krull-Schmidt triangulated category with the co-heart C of a bounded co-t-structure. By [26, Corollary 5.9], there is a natural bijection between bounded cohearts of $D$ and silting subcategories $C$, i.e. $\left.\operatorname{Hom}^{>0}(C, C)\right)=0$ and $\operatorname{thick}_{D}(C)=D$. If $C$ has an additive generator, i.e. $\mathrm{C}=\operatorname{add} C$ for some $C \in \mathrm{D}$, then $C$ is a silting object.

By [26], see also [2], the bounded co-t-structure $\left(\mathrm{X}_{\mathbf{C}}, \mathbf{Y}_{\mathbf{C}}\right)$ can be recovered from the co-heart C using the formulas

$$
\mathrm{X}_{\mathrm{C}}=\bigcup_{k>0} \Sigma^{-k} \mathrm{C} * \Sigma^{-k+1} \mathrm{C} * \cdots * \Sigma^{-1} \mathrm{C} \quad \text { and } \quad \mathrm{Y}_{\mathrm{C}}=\bigcup_{k \geq 0} \mathrm{C} * \Sigma \mathrm{C} * \cdots * \Sigma^{k} \mathrm{C}
$$


For integers $p \leq q$ we set $\mathrm{C}^{p, q}:=\Sigma^{p} \mathrm{C} * \Sigma^{p+1} \mathrm{C} * \cdots * \Sigma^{q-1} \mathrm{C} * \Sigma^{q} \mathrm{C}$.

Let $D \in \mathrm{D}$. If $D \in \Sigma^{m} \mathrm{C}^{p, q}$ for some $m, p, q \in \mathbb{Z}$ we shall say that $D$ is $(q-p)$-term with respect to $\mathrm{C}$.

The above formulas for $X_{C}$ and $Y_{C}$ boil down to the observation that given a bounded co-t-structure with co-heart $\mathrm{C}$, each object $0 \neq D \in \mathrm{D}$ admits a Postnikov tower

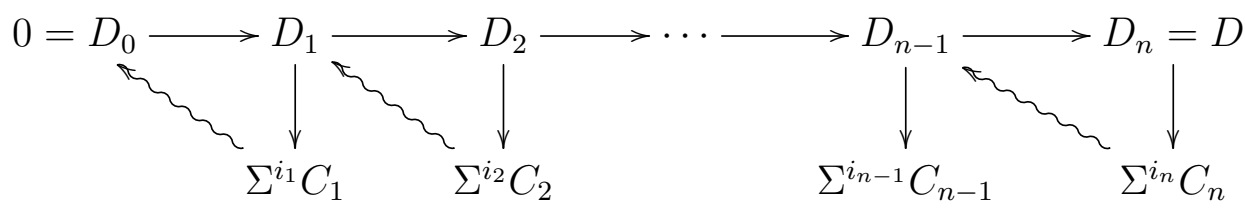

with $i_{1}<i_{2}<\cdots<i_{n}$ and $C_{i} \in \mathrm{C}$; see [12, Proposition 1.5.6].

In analogy with bounded t-structures, there is a canonical isomorphism of groups $K_{0}^{\text {split }}(\mathrm{C})=K_{0}(\mathrm{D})$, induced by the inclusion $\mathrm{C} \hookrightarrow \mathrm{D}$; see [12, Theorem 5.3.1]. Note that unlike for bounded hearts, we use the split Grothendieck group of the co-heart.

Definition 4.1. Let $(D, C)$ be a triangulated category with a co-heart $C$ of a bounded co-t-structure. We call $(\mathrm{D}, \mathrm{C})$ discrete with respect to $\mathrm{C}$, or $\mathrm{C}$-discrete, if for each group valued function $v: \mathbb{Z} \rightarrow K_{0}(\mathrm{D})$ the following set of objects is finite:

$\left\{D \in \mathrm{D} \mid D\right.$ admits a filtration $(1) \operatorname{such}$ that $\operatorname{supp}(v)=\left\{i_{1}, \ldots, i_{n}\right\}$ and $\left.\left[C_{j}\right]=v\left(i_{j}\right)\right\}$.

In contrast to the implications of Theorem 2.5, the following co-t-structure analogue gives an equivalence. To assess this, think of silting subcategories as having the modularity condition "built in"; a concrete instance is the correspondence between silting subcategories and algebraic t-structures for finite-dimensional algebras in [24].

Theorem 4.2. Let $(\mathrm{D}, \mathrm{C})$ be a triangulated category together with the co-heart $\mathrm{C}=\operatorname{add} C$ of a bounded co-t-structure. Then

$$
\mathrm{D} \text { is cone-finite } \Longleftrightarrow \mathrm{D} \text { is discrete with respect to } \mathrm{C} \text {. }
$$

Proof. $(\Longrightarrow)$ The proof is essentially the same as the proof of Theorem 2.5(i), where we instead write for a function $v: \mathbb{Z} \rightarrow K_{0}(\mathrm{D})$,

$$
\mathrm{D}_{v}:=\left\{A \in \mathrm{D} \mid A \text { admits a filtration (1) with } \operatorname{supp}(v)=\left\{i_{1}, \ldots, i_{n}\right\} \text { and }\left[C_{j}\right]=v\left(i_{j}\right)\right\} \text {. }
$$

The only part where the proof differs is the base step of the induction, i.e. length $(v)=0$. Again, without loss of generality we may assume $v(0) \neq 0$. Since $\mathrm{C}=\operatorname{add} C$, where $C=C_{1} \oplus \cdots \oplus C_{n}$ say, is a silting object, each object $C^{\prime} \in \mathrm{C}$ decomposes uniquely as $C^{\prime}=C_{1}^{m_{1}} \oplus C_{2}^{m_{2}} \oplus \cdots \oplus C_{n}^{m_{n}}$, whence $\left[C^{\prime}\right]=m_{1}\left[C_{1}\right]+\cdots+m_{n}\left[C_{n}\right]$. Therefore, the class of $\left[C^{\prime}\right] \in K_{0}^{\text {split }}(\mathrm{C})$ is uniquely determined by its Krull-Schmidt decomposition. This says, in particular, that $\mathrm{D}_{v}$ is a singleton when length $(v)=0$. The remainder of the proof proceeds as in Theorem 2.5(i), noting that the uniqueness of the decomposition triangle $A^{\prime} \rightarrow A \rightarrow A^{\prime \prime} \rightarrow \Sigma A^{\prime}$ is not required for the proof to work.

$(\Longleftarrow)$ Let $A, B \in \mathrm{D}$. We want to show that the set $\mathrm{Z}:=\{Z \mid \exists A \stackrel{f}{\rightarrow} B \rightarrow Z \rightarrow \Sigma A\}$ is finite. We proceed in two steps.

Step 1: $A$ is 1-term with respect to $C$ and $B$ is $n$-term with respect to $C$, for some $n \geq 1$.

Without loss of generality we may assume that $A=\Sigma^{m} C$ for some $C \in \mathrm{C}$ and some $m \in \mathbb{Z}$ and $B \in \mathrm{C}^{0, n}$. In particular, this means that $B$ admits a filtration,

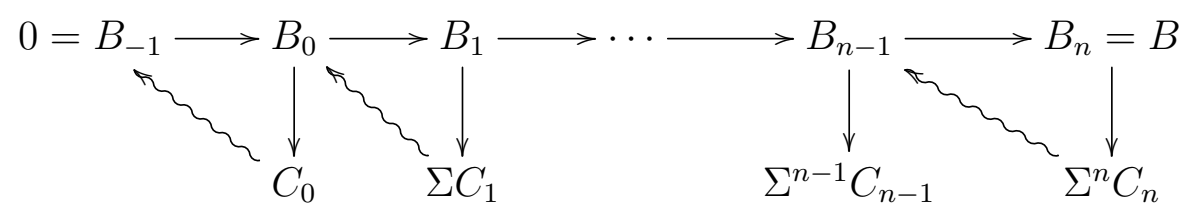


with the $C_{i} \in \mathrm{C}$, some of them possibly zero. We consider various possibilities for $m$.

If $m<0$ then $\operatorname{Hom}(A, B)=0$ and $\mathbf{Z}$ is trivially finite.

If $m \geq n$, then we get the following filtration for any $Z \in \mathrm{Z}$,

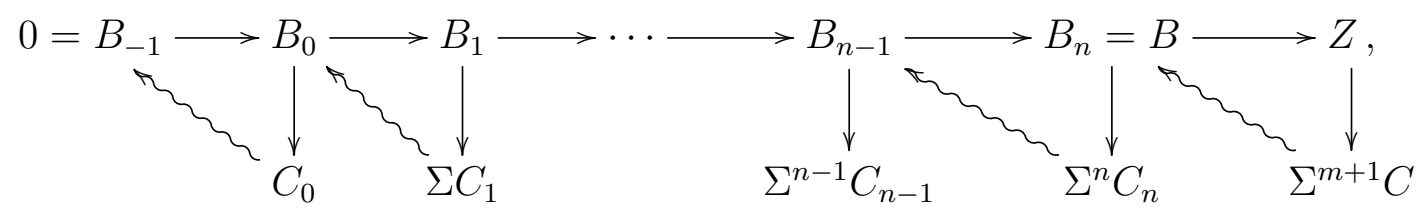

whence by $C$-discreteness there are only finitely many $Z$ admitting a filtration with these filtrands, making $\mathrm{Z}$ finite.

If $0 \leq m<n$, we consider the diagram coming from the octahedral axiom.

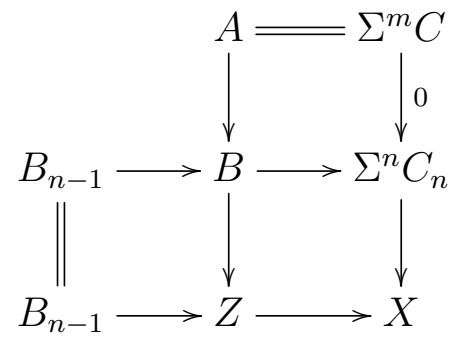

Thus, $X=\Sigma^{n} C_{n} \oplus \Sigma^{m+1} C$. If $m=n-1$ then we get the filtration:

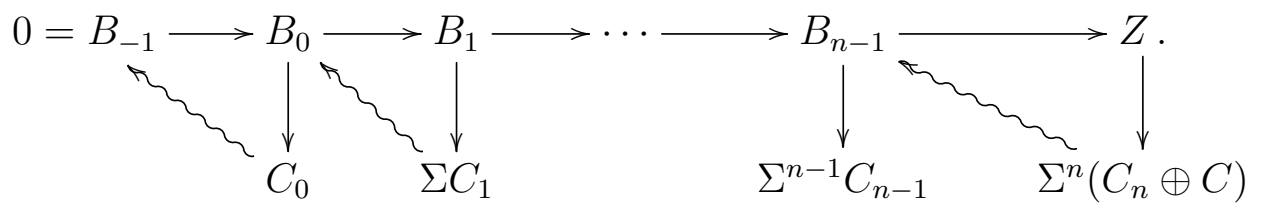

Otherwise, using [21, Lemmas 7.1 and 7.2$]$ in sequence gives the filtration

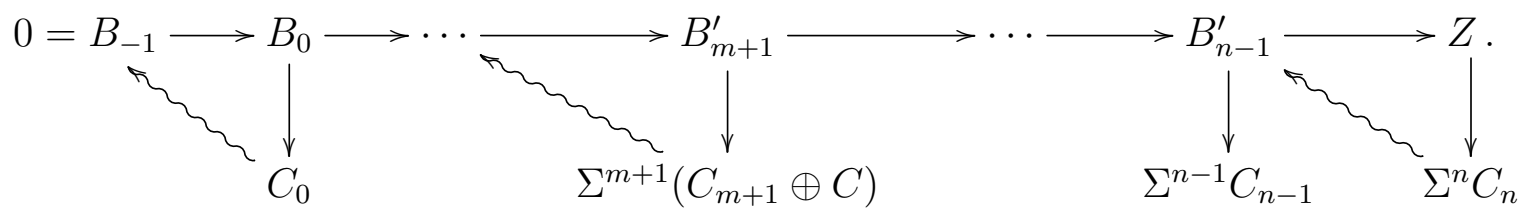

In either case, $\mathbf{C}$-discreteness affirms the finiteness of $\mathbf{Z}$.

Step 2: Both $A$ and $B$ are of arbitrary length with respect to $\mathrm{C}$.

We proceed by induction on the length of $A$ with respect to C; cf. proof of Theorem 1.2. Suppose $A$ is $n$-term with respect to $\mathrm{C}$. Then $A$ admits a decomposition $A^{\prime} \rightarrow A \rightarrow$ $A^{\prime \prime} \rightarrow \Sigma A^{\prime}$ in which $A^{\prime}$ is $(n-1)$-term and $A^{\prime \prime}$ is 1 -term with respect to C. Now consider the diagram coming from the octahedral axiom:

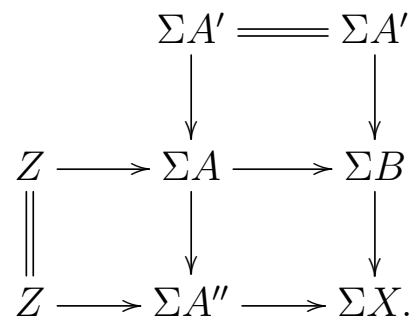

By induction, there are finitely many possible $\Sigma X$, whence by Step 1, and the fact that $A^{\prime \prime}$ is 1 -term with respect to $\mathrm{C}$, there are finitely many possible $Z$.

Corollary 4.3. If $\mathrm{D}$ is discrete with respect to a silting subcategory $\mathrm{C}$ of $\mathrm{D}$, then $\mathrm{D}$ is discrete with respect to any other silting subcategory $\mathrm{C}^{\prime}$. 
For the next corollary, we remark that $\mathrm{D}^{b}(\bmod (\Lambda))$ has a natural bounded t-structure, with heart $\bmod (\Lambda)$, and $\mathrm{K}^{b}(\operatorname{proj}(\Lambda))$ has a natural co-t-structure, with co-heart $\operatorname{proj}(\Lambda)$. However, by Proposition 3.2 and the previous corollary, the actual choices of heart and co-heart do not matter.

Corollary 4.4. Let $\Lambda$ be a finite-dimensional $\mathbf{k}$-algebra. If $\mathrm{D}^{b}(\bmod (\Lambda))$ is discrete with respect to $\bmod (\Lambda)$ then $\mathrm{K}^{b}(\operatorname{proj}(\Lambda))$ is discrete with respect to $\operatorname{proj}(\Lambda)$.

Proof. $\mathrm{D}^{b}(\bmod (\Lambda))$ is cone finite by Theorem 2.5 as $\bmod (\Lambda)$ is modular. Hence the full subcategory of $\mathrm{K}^{b}(\operatorname{proj}(\Lambda)) \subseteq \mathrm{D}^{b}(\bmod (\Lambda))$ is also cone finite. Whence Theorem 4.2 implies $\mathrm{K}^{b}(\operatorname{proj}(\Lambda))$ is discrete with respect to $\operatorname{proj}(\Lambda)$.

Remark 4.5. Vossieck's main result $\left[28, \S 2\right.$, Theorem] asserts that $\mathrm{D}^{b}(\bmod (\Lambda))$ is discrete if and only if $\mathrm{K}^{b}(\operatorname{proj}(\Lambda))$ is discrete (taking homology with respect to $\bmod (\Lambda)$ ).

However, in general, there is no intrinsic definition of discreteness in $\mathrm{K}^{b}(\operatorname{proj}(\Lambda))$. For example, $\mathrm{D}^{b}\left(\bmod \left(\mathbf{k}[x] /\left(x^{2}\right)\right)\right)$ is discrete with respect to $\bmod (\Lambda)$, but $\mathrm{K}^{b}\left(\operatorname{proj}\left(\mathbf{k}[x] /\left(x^{2}\right)\right)\right)$ has no bounded t-structure [20] so that the discreteness notion of [28] does not apply. Nevertheless, $\mathbf{K}^{b}\left(\operatorname{proj}\left(\mathbf{k}[x] /\left(x^{2}\right)\right)\right)=\mathbf{K}^{b}(\operatorname{proj}(\Lambda(1,1,0)))$ is discrete with respect to $\operatorname{proj}(\Lambda)$ by Corollary 4.4, and more generally, the same holds for all $\mathrm{K}^{b}(\operatorname{proj}(\Lambda(n, n, m)))$.

\section{ExAmples}

In the following table, we present some triangulated categories exhibiting interesting behaviour with regards to the various smallness notions studied in this article. For the convenience of the reader, we briefly summarise these notions:

$\mathrm{H}$-discrete: There is a bounded heart $\mathrm{H}$ such that for any $v: \mathbb{Z} \rightarrow K_{0}(\mathrm{H})$, the set of objects $D \in \mathrm{D}$ with $\left[H^{i}(D)\right]=v(i)$ for all $i$ is finite. In all example classes below, if this property holds for one bounded heart, it holds for all.

C-discrete: There is a silting subcategory $\mathrm{C}$ such that for any $v: \mathbb{Z} \rightarrow K_{0}^{\text {split }}(\mathrm{C})$, the set of objects admitting a Postnikov tower having filtrands $\Sigma^{i_{j}} C_{j}$ with $i_{1}<\cdots<i_{n}$ and $C_{i} \in \mathrm{C}$ is finite.

hom bound: There is a universal bound on Hom dimensions among indecomposable objects; the subscript indicates the maximal bound occurring in the family.

cone finite: Any two objects admit only finitely many cones, up to isomorphism.

finite hearts: Hearts of bounded t-structures have finitely many indecomposables.

discrete hearts: Any object $H \in \mathrm{H}$ of any bounded heart is determined up to finite ambiguity by $[H] \in K_{0}(\mathrm{H})$.

countable: The category has only countably many objects, up to isomorphism.

Several of these properties make no sense for triangulated categories without bounded (co-)t-structures. This is indicated by - in the table. The examples assume that $\mathbf{k}$ is an uncountable field.

\begin{tabular}{lccccccccccc}
\hline & $\mathrm{k} \Gamma_{\mathrm{ADE}}$ & $\mathrm{k} A_{\infty}$ & $\mathrm{DDC}$ & $\mathrm{DDC}^{c}$ & $\mathcal{C}_{\mathrm{ADE}}$ & $\mathrm{T}_{1}$ & $\mathrm{~T}_{>1}$ & $\mathrm{~T}_{<1}$ & $\mathrm{~T}_{1, n}$ & $\mathbb{Q} \tilde{A}_{1}$ & $\mathbb{F}_{q} \tilde{A}_{1}$ \\
\hline H-discrete & $\checkmark$ & $\checkmark$ & $\checkmark$ & - & - & $\checkmark$ & $\checkmark$ & - & - & $\times$ & $\checkmark$ \\
C-discrete & $\checkmark$ & $\checkmark$ & $\checkmark$ & $\checkmark$ & - & - & - & $\checkmark$ & - & $\times$ & $\checkmark$ \\
hom bounded & $\checkmark$ & $\checkmark_{1}$ & $\checkmark$ & $\checkmark$ & $\checkmark$ & $\checkmark$ & $\times$ & $\checkmark$ & $\checkmark_{2}$ & $\checkmark_{\lfloor n / 2\rfloor} \times$ & $\times$ \\
cone finite & $\checkmark$ & $\checkmark$ & $\checkmark$ & $\checkmark$ & $\checkmark$ & $\checkmark$ & $\checkmark$ & $\checkmark$ & $\checkmark$ & $\times$ & $\checkmark$ \\
finite hearts & $\checkmark$ & $\times$ & $\checkmark$ & - & - & $\times$ & $\checkmark$ & - & - & $\times$ & $\times$ \\
discrete hearts & $\checkmark$ & $\checkmark$ & $\checkmark$ & - & - & $\checkmark$ & $\checkmark$ & - & - & $\times$ & $\checkmark$ \\
countable objects & $\checkmark$ & $\checkmark$ & $\checkmark$ & $\checkmark$ & $\checkmark$ & $\checkmark$ & $\checkmark$ & $\checkmark$ & $\checkmark$ & $\checkmark$ & $\checkmark$ \\
\hline
\end{tabular}


We proceed to explain the example classes.

$\mathrm{k} \Gamma_{\mathrm{ADE}}, \mathrm{k} A_{\infty}$ - quiver algebras: By listing finite-dimensional algebras, we mean their bounded derived categories.

$\Gamma_{\mathrm{ADE}}$ stands for an ADE quiver, so that the corresponding algebra is hereditary and representation-finite. The maximal hom bound of 6 is achieved in type $E_{8}$.

$A_{\infty}$ stands for the (one-sided) infinite, zigzag oriented quiver of type $A$. This example is interesting because $\mathrm{D}^{b}\left(\mathbf{k} A_{\infty}\right)$ is hom bounded, but has infinite hearts; note it does not have a classical generator.

DDC, $\mathrm{DDC}^{c}$ - derived-discrete algebras: DDC is a shorthand for $\mathrm{D}^{b}(\Lambda(r, n, m))$, the derived-discrete category for the algebra with $r$ consecutive relations in an $n$-cycle and a tail of length $m$; see Section 3. We assume $r<n$, so that AR triangles (i.e. a Serre functor) exist. Note that $\mathrm{D}^{b}(\Lambda(n, n, m))$ has no bounded co-t-structures.

DDC ${ }^{c}$ stands for $\mathrm{K}^{b}(\operatorname{proj}(\Lambda(n, n, m)))$, the bounded homotopy category of projective modules over a derived-discrete algebra of infinite global dimension. This is both the subcategory of perfect complexes of $\mathrm{D}^{b}(\Lambda(n, n, m))$ and the the subcategory of compact objects in $\mathrm{D}(\Lambda(n, n, m))$. These algebras are gentle, hence Gorenstein, so that $\mathrm{DDC}^{c}$ has AR triangles [17].

$\mathcal{C}_{\mathrm{ADE}}$ - cluster categories: $\mathcal{C}_{\mathrm{ADE}}=\mathrm{D}^{b}\left(\mathrm{k} \Gamma_{\mathrm{ADE}}\right) / \Sigma^{-1} \tau$ stands for the cluster category of type ADE, where $\tau$ is the Auslander-Reiten translation. It is triangulated by [23] and has finitely many indecomposables.

$\mathrm{T}_{1}, \mathrm{~T}_{>1}, \mathrm{~T}_{<1}$ - spherical generators: For $w \in \mathbb{Z}$, let $\mathrm{T}_{w}$ be the triangulated category generated by a $w$-spherical object, i.e. an object whose derived endomorphism algebra is $\mathbf{k} \oplus \Sigma^{-w} \mathbf{k}$. Note that $T_{1}$ is the bounded derived category of the hereditary standard homogeneous tube. Hom bounds for $T_{<0}$ are 1 , and for $T_{0}$ it is 2 .

The categories $T_{<1}$ have no bounded t-structures [20], making it pointless to ask for $\mathrm{H}$-discreteness or finite hearts. Likewise, $\mathrm{T}_{\geq 1}$ has no bounded co-t-structures.

$\mathrm{T}_{1, n}$ - truncated tubes: For $n>1$, we let $\mathrm{T}_{1, n}=\underline{\bmod }\left(\mathbf{k}[x] /\left(x^{n}\right)\right)$ be the stable module category, e.g. $\mathbf{k}[x] /\left(x^{2}\right)=\Lambda(1,1,0)$. The AR quiver of $\mathrm{T}_{1, n}$ is the following truncated homogeneous tube:

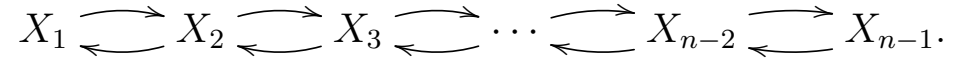

The unique projective module $X_{n}$ does not occur because we have taken the stable category. The algebra is selfinjective, hence $T_{1, n}$ is triangulated. As $T_{1, n}$ has only $n-1$ many indecomposable objects and is Krull-Schmidt, it is cone finite. Moreover, from $\operatorname{dim} \underline{\operatorname{Hom}}\left(X_{i}, X_{i}\right)=\min \{i, n-i\}$, we see that arbitrary hom bounds can be attained. Note that $T_{1, n}$ has no bounded t-structures.

Small fields: $\tilde{A}_{1}$ is the Kronecker quiver, and the last two columns denote $\mathrm{D}^{b}\left(\mathbb{Q} \tilde{A}_{1}\right)$ and $\mathbf{D}^{b}\left(\mathbb{F}_{q} \tilde{A}_{1}\right)$, respectively. Instead of $\mathbb{Q}$, any infinite countable field works. By Remark 1.3 , we could replace $\tilde{A}_{1}$ by any finite-dimensional algebra. We chose the Kronecker quiver because it is manifestly non-discrete for uncountable fields.

\section{REFERENCES}

[1] T. Adachi, Y. Mizuno, D. Yang: Silting-discrete triangulated categories and contractible stability spaces, arXiv: 1708.08168.

[2] T. Aihara, O. Iyama: Silting mutation in triangulated categories, J. Lond. Math. Soc. (2) 85 (2012), 633-668, also arXiv:1009.3370.

[3] K.K. Arnesen, R. Laking, D. Pauksztello: Morphisms between indecomposable complexes in the bounded derived category of a gentle algebra, J. Algebra 467 (2016), 1-46, also arXiv:1411.7644. 
[4] K.K. Arnesen, R. Laking, D. Pauksztello, M. Prest: The Ziegler spectrum of a derived-discrete algebra, Adv. Math. 319 (2017), 653-698, also arXiv:1603.00775.

[5] I. Assem, D. Simson, A. Skowroński: Elements of the representation theory of associative algebras. 1: Techniques of representation theory, London Math. Soc. Stud. Texts, vol 65, Cambridge University Press (2006).

[6] M.F. Atiyah: On the Krull-Schmidt theorem with application to sheaves, Bull. Soc. Math. France 84 (1956), 307-317.

[7] V. Bekkert, H.A. Merklen: Indecomposables in derived categories of gentle algebras, Algebr. Represent. Theory 6(3) (2003), 285-302.

[8] A. Beligiannis: Relative homological algebra and purity in triangulated categories, J. Algebra 227 (2000), 268-361.

[9] G. Bobiński: The almost split triangles for perfect complexes over gentle algebras, J. Pure Appl. Algebra 215(4) (2011), 642-654, also arXiv:0903.5140.

[10] G. Bobiński, C. Geiß, A. Skowroński: Classification of discrete derived categories, Central Eur. J. Math. 2 (2004), 19-49.

[11] G. Bobiński, H. Krause: The Krull-Gabriel dimension of discrete derived categories, Bull. Sci. Math. 139 (2015), no. 3, 269-282, also arXiv:1402.1596.

[12] M.V. Bondarko: Weight structures vs. t-structures; weight filtrations, spectral sequences, and complexes (for motives and in general), J. K-Theory 6 (2010), 387-504, also arXiv:0704.4003.

[13] N. Broomhead, D. Pauksztello, D. Ploog: Discrete derived categories I: Homomorphisms, autoequivalences and t-structures, Math. Z. 185(1) (2017), 39-89, also arXiv:1312.5203.

[14] N. Broomhead, D. Pauksztello, D. Ploog: Discrete derived categories II: The silting pairs CW complex and the stability manifold, J. London Math. Soc. (2) 93 (2016), no. 2, 273-300, also arXiv: 1407.5944.

[15] I. Çanakçi, D. Pauksztello, S. Schroll: On extensions for gentle algebras, arXiv:1707.06934.

[16] G. Garkusha, M. Prest: Triangulated categories and the Ziegler spectrum, Algebr. Represent. Theory 8 (2005), no. 4, 499-523.

[17] C. Geiss, I. Reiten: Gentle algebras are Gorenstein, in Representations of algebras and related topics, Fields Institute Communications 45 (Amer. Math. Soc., Providence, RI, 2005), 129-133.

[18] Y. Han, C. Zhang: Brauer-Thrall type theorems for derived module categories, Algebr. Represent. Theory 19 (2016), no. 6, 1369-1386, also arXiv:1310.2777.

[19] Z. Han: A note on generic objects and locally finite triangulated categories, Appl. Categ. Structures 24 (2016), no. 6, 875-884, also arXiv:1412.1159.

[20] T. Holm, P. Jørgensen, D. Yang: Sparseness of t-structures and negative Calabi-Yau dimension in triangulated categories generated by a spherical object, Bull. London Math. Soc. 45 (2013), 120-130, also arXiv:1108.2195.

[21] P. Jørgensen, D. Pauksztello: The co-stability manifold of a triangulated category, Glasg. Math. J. 55 (2013), 161-175, also arXiv:1109.4006.

[22] M. Kashiwara, P. Schapira: Sheaves on manifolds, Grundlehren der Mathematischen Wissenschaften 292, Springer, Berlin, 1994.

[23] B. Keller: On triangulated orbit categories, Doc. Math. 10 (2005), 551-581, also arXiv:math/0503240.

[24] S. König, D. Yang: Silting objects, simple-minded collections, t-structures and co-t-structures for finite-dimensional algebras, Doc. Math. 19 (2014), 403-438, also arXiv:1203.5657.

[25] H. Krause: Cohomological length functions, Nagoya Math. J. 223 (2016), no. 1, 136-161, also arXiv: 1209.0540

[26] O. Mendoza Hernández, E.C. Sáenz Valadez, V. Santiago Vargas, M.J. Souto Salorio: Auslander-Buchweitz context and co-t-structures, Appl. Categ. Structures 21 (2013), 119-139, also arXiv: 1002.4604

[27] D. Pauksztello, M. Saorín, A. Zvonareva: Contractibility of the stability manifold for silting discrete algebras, arXiv:1705.10604.

[28] D. Vossieck: The algebras with discrete derived category, J. Algebra 243 (2001), 168-176.

Email: nathan.broomhead@plymouth.ac.uk, d.pauksztello@lancaster.ac.uk, dploog@math.fu-berlin.de Nathan Broomhead, Mathematical Sciences, Plymouth University, Drake Circus, Plymouth, PL4 8AA, United Kingdom David Pauksztello, Department of Mathematics and Statistics, Lancaster University, Lancaster, LA1 4YF, United Kingdom David Ploog, Arnimallee 3, Mathematisches Institut, Freie Universität Berlin, 14195 Berlin, Germany 